\title{
Biodiversity and antibacterial activity of endophytic fungi isolated from jambu bol (Syzygium malaccense)
}

\author{
YUSTINA HAPIDA $^{1,2, \bullet}$, ELFITA $^{3, \bullet \bullet}$, HARY WIDJAJANTI ${ }^{4}$, SALNI $^{4}$ \\ ${ }^{1}$ Graduate School of Sciences, Faculty of Mathematics and Natural Sciences, Universitas Sriwijaya. J1. Padang Selasa No. 524, Palembang 30128, South \\ Sumatra, Indonesia \\ ${ }^{2}$ Universitas Islam Negeri Raden Fatah. Jl. Prof. K. H. Zainal Abidin Fikri Km 3, Pahlawan, Kemuning, Palembang 30126, South Sumatra, Indonesia. \\ "email: yustinahapida_uin@ @radenfatah.ac.id \\ ${ }^{3}$ Department of Chemistry, Faculty of Mathematics and Natural Sciences, Universitas Sriwijaya. Jl. Palembang-Prabumulih Km. 32, Ogan Ilir 30862, \\ South Sumatra, Indonesia. Tel.: +62-711-580056, Fax.: +62-711-580268, •"email: elfita69@gmail.com \\ ${ }^{4}$ Department of Biology, Faculty of Mathematics and Natural Sciences, Universitas Sriwijaya. Jl. Palembang-Prabumulih Km. 32, Ogan Ilir 30862, South \\ Sumatra, Indonesia
}

Manuscript received: 24 October 2021. Revision accepted: 29 November 2021.

\begin{abstract}
Hapida Y, Elfita, Widjajanti H, Salni. 2021. Biodiversity and antibacterial activity of endophytic fungi isolated from jambu bol (Syzygium malaccense). Biodiversitas 22: 5668-5677. Infectious diseases caused by pathogenic bacteria are still high. The existence of bacterial resistance to antibiotics makes it necessary to search for new antibacterial substances from plants and microorganisms. Syzygium malaccense (L.) Merr and Perry (Myrtaceae), known as jambu bol, has been used as a traditional medicine in Indonesia and other countries. It is used to treat pathogenic bacterial infections. Therefore, this study aims to determine the diversity of endophytic fungi and their potential as antibacterial compound sources compared to their host plants. Specifically, screening for endophytic fungi that have antibacterial activities is intended. In this study, endophytic fungi were isolated from the leaves, stem bark, and root bark of the host plants. Each isolate was identified morphologically and cultivated in potato dextrose broth (PDB) medium for four weeks. After the incubation period, the liquid culture was extracted with ethyl acetate and evaporated to obtain a concentrated extract. The KirbyBauer method was used to evaluate the antibacterial activity of each endophytic fungal extract and the host plant parts extract. The concentration used was $400 \mu \mathrm{g} / \mathrm{mL}$ and the antibiotic was used as a positive control (Tetracycline) is $30 \mu \mathrm{g} / \mathrm{mL}$. Seven endophytic fungi were isolated from the leaves (YD1, YD2, YD3, YD4, YD6, YD7, Y2D1), seven from the stem bark (YB1, YB2, YB3, YB4, YB5, Y2B5, and Y2B6 ), and six from the root bark (YA1, YA2, YA3, YA4, YA5, Y2A2). The endophytic fungi were morphologically identified, and the isolates were shown to be dominated by genera from the Ascomycota (e.g., Poaceascoma, Cladorrhinum, Penicillium, Madurella, Phytophthora, Phialemonium, Monascus, Trichoderma, Ramophialophora, Gliocladium, Wiesneriomyces) and the Zygomycota (e.g., Mucor, Mortierella, and Gongronella) phyla. The fungi isolates Y2D1, YB1, and YA4 were shown to have the highest (strong) antibacterial activity equivalent to their hosts in the performed screening. Endophytic fungi have significant and continued antibacterial activities that have been identified molecularly.
\end{abstract}

Keywords: Antibacterial, endophytic fungi, Syzygium malaccese

\section{INTRODUCTION}

Plant specimens from the Syzygium genera, in which the jambu bol plants (Syzygium malaccense (L.) Merr and Perry (Myrtaceae) included, used by people in South Sumatra as a traditional medicine for various diseases, including pathogenic bacterial infections (Sonavane 2017; Fernandes and Rodrigues 2018). According to the results found in the literature, jambu bol plants have been used by people in other countries for treating various diseases in traditional medicine. In Brazil, jambu bol is used to treat diabetes (Freitas et al. 2015). In India, some parts of the plant such as fruit, seeds and bark are used in treating dysentery, diabetes, and gastric ulcers (Bairy et al. 2005). In Nigeria, these plants are used to treat diabetes, antiinflammatory, and antimicrobial activities (Oyinlade 2014). Leaf extract was shown to be a growth inhibitor of Staphylococcus aureus and Streptococcus pyogenes at a $100 \mathrm{~g} / \mathrm{mL}$ concentration (Locher et al. 1995). Some compounds from S. malaccense leaves and bark (e.g.,
5,7,3', 4', 5'-pentahydroxy flavonol compounds; 5,7,3',5'tetra-hydroxy-4'-methoxy flavonol; 5-trihydroxybenzoic acid; 3-acetyl-urs-12-en-28-oic acid; and 3 $\beta$-O-acetyl-urs12-en-28-oic acid) and the napin peptide from the seeds were shown to be new potential anti-Staphylococcus substances ( da Silva Dantas et al. 2014).

Plants with ethnobotanical historical use in treating certain diseases are promising candidates for obtaining bioactive compounds to control high-activity endophytic fungi (Strobel and Daisy 2003; Abdalla and McGaw 2018). The current prospect of bioactive compounds and the high demand for medicinal ingredients have spurred the search for new bioactive metabolites inside medicinal plant endophytic fungi (Zhao et al. 2010). Endophytic fungi are promising and abundant sources of natural products with varied chemical structures, significant biodiversity, and many exciting bioactivities (Manganyi and Ateba 2020; ElBondkly et al. 2021). Natural products derived from endophytic fungi are considered the most relevant sources for discovering new molecular drug (Singh et al. 2021). It 
was indicated in several studies that most bioactive metabolites obtained in endophytic fungi carry unique chemical structures. These fungi can produce metabolites similar to those produced by their host plants. Still, different new compounds have been discovered (Cruz et al. 2020; El-Hawary et al. 2020). The diversity of endophytic fungi obtained will be useful as an alternative to replace the supply shortage of plant resources, shorten the regeneration cycle, and use industrial fermentation to produce natural active compounds, such as antimicrobial and other bioactive compounds, for mass production at a low cost and without pollution through biotechnology, so its potential still needs to be explored (Du et al. 2020)

This scenario represents a significant biotechnological potential for the discovery of new drugs in microbial groups. Research regarding the antibacterial pharmacological effects of bioactive compounds obtained from the endophytic fungi hosted by $S$. malaccense is still in its initial stages. Nevertheless, by reviewing the documentation of endophytic fungal diversity, new insights into the possibilities for exploration and development may be provided. Therefore, the classification of pharmacological functions from extracts obtained from endophytic fungi hosted by $S$. malaccense specimens may be assisted by antibacterial pharmacological assays. With such assays, bioprospection information of endophytic fungi as biological entities may be provided, ensuring their continued use to improve human quality of life.

\section{MATERIALS AND METHODS}

\section{Plant material}

Samples from leaves, stem bark, and root bark of healthy $S$. malaccense plants were obtained in Palembang, South Sumatra, Indonesia in February 2021. This plant was identified by the Indonesian Institute of Sciences (LIPI) Plant Conservation Center, Botanical Gardens Purwodadi, East Java, Indonesia with no: B-302/III//KS. 01.03/1/2021.

\section{Isolation of endophytic fungi}

The surface of the plant samples (leaves, stem bark and root bark) was sterilized, later, they were cut longitudinally using an aseptic technique. Additionally, the samples were included in a petri dish containing PDA (Potato dextrose agar) medium. Incubation was conducted at $37^{\circ} \mathrm{C}$ for $3-10$ days. Observations were made every day until the fungus was able to be seen. Fungal colonies growing on PDA (Potato Dextrosa Agar) medium showed different morphological that it was purified. Observations were made every day until it was possible to see the fungus. Fungal colonies growing on PDA medium had different morphological features, indicating that the samples were purified (Fadhillah et al. 2019).

\section{Morphological identification of endophytic fungi}

The identification of fungi was conducted based on three main characteristics: colonies, microscopic characteristics, and macroscopic characteristics. Macroscopic identification of endophytic fungi included observations on surface and reverse colonies, pattern, texture, topography, consentric and radiated circle. Microscopic identification by observing hypae, spore type, shape of spores and other specific characteristics by making culture slides first and the medium used is PDA (Pitt and Hocking 2009; Watanabe 2010; Walsh et al. 2018).

\section{Cultivation and extraction}

Each endophytic fungi was isolated during cultivation. Six pieces of PDA media (approximately $5 \mathrm{~mm}$ diameter) filled with pure fungal culture were placed into three erlenmeyer flasks containing $300 \mathrm{~mL}$ of potato dextrose broth (PDB) medium. Incubation was conducted at room temperature for approximately four weeks. The medium containing secondary metabolites was partitioned into ethyl acetate and evaporated to obtain a concentrated ethyl acetate extract (Habisukan et al. 2021).

\section{Extraction of the host plant Syzygium malaccense}

Approximately $100 \mathrm{~g}$ of fresh leaves, bark, and root bark of the plants were obtained. The samples were then dried at room temperature and macerated using methanol as a solvent for 24 hours with two repetitions to obtain the extracts. Later, the extract was filtered with filter paper and evaporated with a rotary evaporator (Metasari et al. 2020). Methanol used as a polar solvent more effective in extracting phytochemical compounds in natural materials such as plants to increase their biological activity (Nawaz et al. 2020).

\section{Antibacterial activity test}

An antibacterial activity test was performed using the disc diffusion method, also known as Kirby-Bauer, in a petri dish containing NA (Nutrien Agar) medium. The sample fungi extracts were put on standard disc paper as a test sample $(400 \mu \mathrm{g} / \mathrm{mL})$ with standard antibiotics (tetracycline $30 \mu \mathrm{g} / \mathrm{mL}$ ). The test bacteria used were Salmonella typhi (ATCC1408), Escherichia coli (Ina CCB4), Staphylococcus aureus (Ina CCB5), and Bacillus subtilis (Ina CCB4). Observations and measurements of the inhibition zone were conducted for $24 \mathrm{~h}$. The percentage was determined using the following formula (Ding et al. 2019).

$$
\text { weak: } \frac{A}{B} x 100 \%<50 \% \text {; moderat: } 50 \%<\frac{A}{B} x 100 \%<70 \% \text {; strong: } \frac{A}{B} x 100 \%>70 \%
$$

Where: $\mathrm{A}$ is the zone of inhibition $(\mathrm{mm})$ of the test samples and B is the zone of inhibition ( $\mathrm{mm}$ ) of the standard antibiotic samples.

\section{RESULTS AND DISCUSSION}

\section{Isolation of endophytic fungi}

In Figure 1, the isolated fungi have hyphae, which is indicative of endophytic fungi growth. Endophytic fungi were observed growing around the leaves, stem bark, and root bark. Surface sterilization of plants and antibiotics on PDA medium was conducted during the isolation of 
endophytic fungi. Endophytic fungi were observed growing around the sliced leaf, stem bark, and root bark. In observations made three days after media inoculation, it was possible to detect colony differences caused by different fungi, and purification was conducted.

After purification and isolation of the endophytic fungi, 20 fungi isolates were obtained. There were seven isolates from the leaves, seven from the bark, and six from the root bark. In Figure 1, it can be seen that the fungal hyphae indicated the growth of endophytic fungi because they were found around the leaves, stem bark, and root bark after surface sterilization and put of antibiotics on PDA media during the isolation of endophytic fungi.

On the third day after inoculation, there were differences in the fungi colonies, so purification could be conducted. With the purification of the endophytic fungi, 20 isolates were obtained. There were seven isolates on the leaves, seven from the bark, and six from the root bark. Macroscopic and microscopic colonies of endophytic fungi on the leaves, stem bark and root bark of $S$. malaccense are shown in Figures 2. The characteristics of macroscopic and microscopi its endophytic fungi are shown in tables $(1,2$, and 3 ).

\section{Cultivation and extraction}

With the cultivation of endophytic fungi from each part of $S$. malaccense in PDB medium, 30 bottles of $300 \mathrm{~mL}$ each were obtained. From these bottles, $10 \mathrm{~g}$ of ethyl acetate extract was obtained from the leaves, stem bark, and root bark. On leaves: YD1, YD2, YD3, YD4, YD6, YD7 and Y2D1. On the stem bark: YB1, YB2, YB3, YB4, YB5, Y2B5 and Y2B6. On root bark: YA1, YA2, YA3, YA4, YA5, and Y2A2.

\section{Extraction of the host plant Syzygium malaccense}

The samples of fresh plant parts $(100 \mathrm{~g})$ were dried and macerated. Then, methanol was used as a solvent, and the solution was evaporated. With the methanol extract obtained from the leaves, stem bark, and root bark, $8.5 \mathrm{~g}$, $7.4 \mathrm{~g}$, and $6.9 \mathrm{~g}$ of extract were obtained, respectively.

\section{Antibacterial activity test}

Each endophytic fungi extract was evaluated for its antibacterial activity using the Kirby-Bauer method at a concentration of $400 \mu \mathrm{g} / \mathrm{disk}$. The extracts obtained from the host plant parts were also assessed. Antibacterial activity with the different extracts was performed at the same concentration to compare the strength of their antibacterial activity. The standard antibiotic used was tetracycline $(30 \mu \mathrm{g} /$ disk $)$ which had broad-spectrum activity. The measurement of the inhibition zone and its strength is shown in Tables 4, 5, and 6.

Regarding the antibacterial activity test results on four bacteria, the fungal extract Y2D1 and the methanol extract from the leaves of $S$. malaccense were potential inhibitors of bacterial growth, considering strong criteria. Other groups of endophytic fungi isolates, including YD2 and YD3, also inhibited bacterial growth with moderate criteria. Finally, endophytic fungi isolates YD1, YD4, and YD7 can inhibit bacteria with weak criteria. Antibacterial activity was determined by comparing it with tetracycline antibiotics. The isolate YB1 and YB3 endophytic fungal isolates from the stem bark of $S$. malaccense and methanol extract had antibacterial activities with stronger criteria than tetracycline antibiotics. Another group of endophytic fungal isolate extracts, including YB4, YB5, Y2B5, and Y2B6, had antibacterial activities with moderate criteria, while the YB2 isolate extract had weak criteria for antibacterial activity. The methanol extract and isolates of endophytic fungi obtained from the root bark of $S$. malacense, including YA4 and YA5, could inhibit bacteria with strong criteria. The other isolate groups, such as YA1, YA3, and Y2A2, had antibacterial activities with moderate criteria. Isolate YA2 had antibacterial activities with weak criteria. The percentage values of each isolate are shown in Table 2 and Figure 3.

The diversity values of endophytic fungi on the leaves, stem bark, and root bark of $S$. malaccense were determined using PAST 3, as shown in Figure 4 and Table 3. As the dominance value (D) of fungi in the leaves, stem bark, and root bark ranged from 0-0.5, regarding the Simpson index, it is an indication that there are no dominating genera in these plant parts. The Shannon diversity index is in the $1>$ $\mathrm{H}$ '3 range, which is the moderate requirement, and the Simpson diversity index is in the 0.6-1 moderate diversity range (Arzamani et al. 2018).

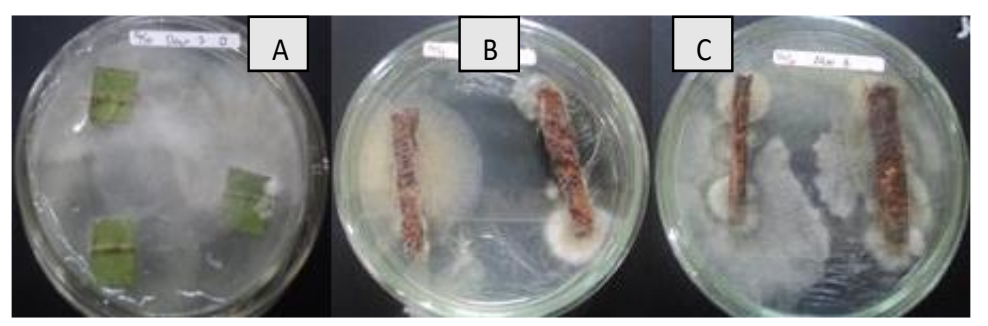

Figure 1. Hyphae from endophytic fungi that began to showed after day 3 around each organ. A) leaves, B) stem bark and C) root bark of jambu bol (Syzygium malaccense) 


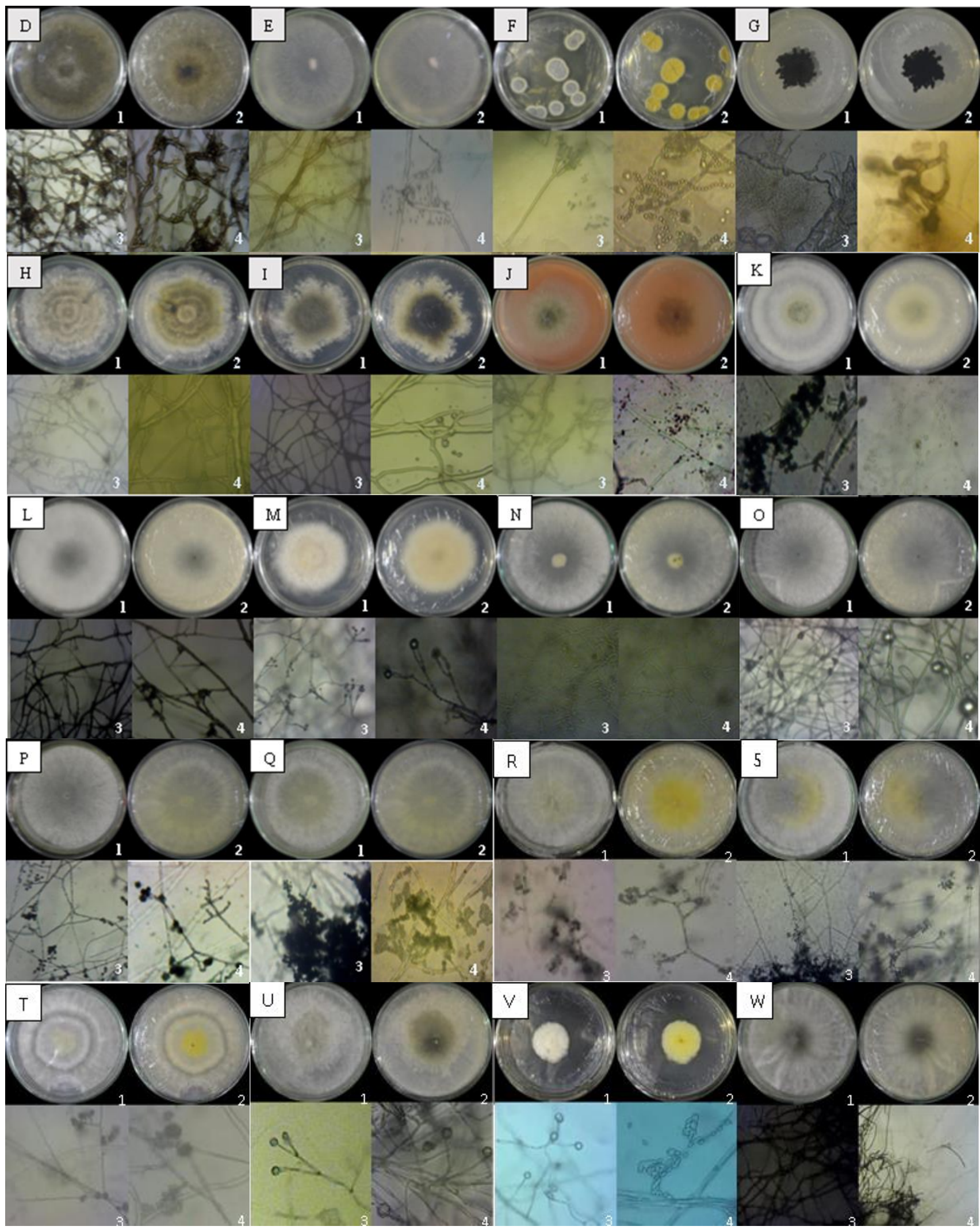

Figure 2. Endophytic fungi isolate on leaf organs, D. YD1, Poaceascoma sp., E. YD2, Cladorrhinum sp., F. YD3, Penicillium sp., G. YD4, Madurella sp., H. YD6, Phytophthora sp., I. YD7, Phialemonium sp., J. Y2D1, Monascus sp., On the stem bark. K. YB1, Trichoderma sp., L. YB2, Ramophialophora sp., M. YB3, Gliocladium sp., N. YB4, Mucor sp., O. YB5, Gonatobotrys sp., P. Y2B5, Trichoderma sp., Q. Y2B6, Trichoderma. On root bark, R. YA1, Trichoderma sp., S. YA2 Trichoderma harzianum. Q. YA3, Trichoderma sp., U. YA4, Mortierella verticillata. V. YA5, Gonatobotrys sp., W. Y2A2, Wiesneriomyces sp. 
Table 1. Macroscopic and microscopic characteristics of the endophytic fungal isolate of the leaf (*), stem bark (**), and root bark (***) Syzygium malaccense

\begin{tabular}{|c|c|c|c|c|c|c|c|c|c|c|}
\hline \multirow[b]{2}{*}{ Isolate } & \multicolumn{5}{|c|}{ Macroscopic characteristics } & \multicolumn{4}{|c|}{ Microscopic characteristics } & \multirow{2}{*}{ Genus / species } \\
\hline & Colony color & $\begin{array}{l}\text { Reverse colony } \\
\text { color }\end{array}$ & Texture & Topography & $\begin{array}{l}\text { Conce } \\
\text { ntric } \\
\text { circle }\end{array}$ & Type of spore & $\begin{array}{l}\text { Shape of } \\
\text { spore }\end{array}$ & Hyphae & Specific characteristic & \\
\hline YD1* & $\begin{array}{l}\text { Yellowish } \\
\text { white }\end{array}$ & $\begin{array}{l}\text { Yellowish } \\
\text { White }\end{array}$ & Cottony & Flat & $\sqrt{ }$ & Klamidospora & Globose & $\begin{array}{l}\text { Septate, } \\
\text { non aerial }\end{array}$ & $\begin{array}{l}\text { Hyaline to dark brown chlamydospores with a thicker } \\
\text { and darkened wall, narrower around septa, and } \\
\text { cylindrical to piriform shape. }\end{array}$ & Poaceascoma sp. \\
\hline YD2* & White & $\begin{array}{l}\text { White, } \\
\text { spreading }\end{array}$ & Cottony & Umbonate & $\sqrt{ }$ & Conidia & Globose & $\begin{array}{l}\text { Septate, } \\
\text { non aerial }\end{array}$ & $\begin{array}{l}\text { Phialides terminal dan lateral berbentuk labu. Konidia } \\
\text { enteroblastik, hialin, berdinding halus dan tipis, bulat. }\end{array}$ & Cladorrhinum sp. \\
\hline YD3* & $\begin{array}{l}\text { Dark green } \\
\text { with marginal } \\
\text { white }\end{array}$ & Light-yellow & Velvety & Rugose & - & Conidia & Globose & $\begin{array}{l}\text { Septate, } \\
\text { aerial }\end{array}$ & $\begin{array}{l}\text { Conidiophores hyaline, erect, branching with } 2-3 \\
\text { metula, each metula has a verticillate phialid, and each } \\
\text { phialid dangles conidia. }\end{array}$ & Penicillium sp. \\
\hline YD4* & Black & Black & Velvety & Rugose & - & Conidia & Globose & $\begin{array}{l}\text { Septate, } \\
\text { non aerial }\end{array}$ & $\begin{array}{l}\text { The hyphae appear to be made up of chains of } \\
\text { spherical cells, indicating that they are budding. There } \\
\text { are also thinner, cylindrical, branching hyphae }\end{array}$ & Madurella sp. \\
\hline YD6* & $\begin{array}{l}\text { White-gray } \\
\text { marginal } \\
\text { yellow }\end{array}$ & Greenish-yellow & Cottony & Flowery & $\sqrt{ }$ & Zoospora & $\begin{array}{l}\text { Obovate, } \\
\text { ellipsoide }\end{array}$ & Septate & $\begin{array}{l}\text { Inside sporangia, zoospores form. Sexual organs do } \\
\text { not develop. Globose hyphae }\end{array}$ & Phytophthora sp. \\
\hline YD7* & $\begin{array}{l}\text { Dark green } \\
\text { marginal white }\end{array}$ & $\begin{array}{l}\text { Dark green } \\
\text { marginal light }\end{array}$ & Cottony & Verrucose & $\sqrt{ }$ & Conidia & Verticillate & $\begin{array}{l}\text { Septate, } \\
\text { aerial }\end{array}$ & $\begin{array}{l}\text { Without a septum at the base to demarcate the hyphae, } \\
\text { phialides grow singly along them. Conidia are hyaline } \\
\text { and single-celled. }\end{array}$ & Phialemonium sp. \\
\hline $\mathrm{Y} 2 \mathrm{D} *$ & $\begin{array}{l}\text { Greenish } \\
\text { white }\end{array}$ & $\begin{array}{l}\text { White greenis } \\
\text { and red pigment } \\
\text { secretion }\end{array}$ & $\begin{array}{l}\text { Cottony } \\
\text { aerial }\end{array}$ & Verrucose & $\sqrt{ }$ & Conidia & Globose & Septate & Dark brown hyphae, branching & Monascus sp. \\
\hline $\begin{array}{l}\text { YB1 } \\
* *\end{array}$ & $\begin{array}{l}\text { Yellowish } \\
\text { white }\end{array}$ & $\begin{array}{l}\text { White, } \\
\text { spreading }\end{array}$ & Cottony & Flat & $\sqrt{ }$ & Conidia & $\begin{array}{l}\text { Sub- } \\
\text { globose }\end{array}$ & $\begin{array}{l}\text { Septate, } \\
\text { aerial }\end{array}$ & $\begin{array}{l}\text { Hyaline conidiophores, have an apical spore mass in } \\
\text { phialides. Phyalophorous conidia. Chlamydospores }\end{array}$ & Trichoderma sp. \\
\hline $\begin{array}{l}\text { YB2 } \\
* *\end{array}$ & $\begin{array}{l}\text { White } \\
\text { Dark centred }\end{array}$ & $\begin{array}{l}\text { White, } \\
\text { spreading }\end{array}$ & Cottony & Flat & $\sqrt{ }$ & Conidia & Globose & $\begin{array}{l}\text { Septate, } \\
\text { non aerial }\end{array}$ & $\begin{array}{l}\text { Conidiophores are reduced to conidiogen cells. Lateral } \\
\text { or terminal phialides of aerial mycelia. }\end{array}$ & $\begin{array}{l}\text { Ramophialophora } \\
\text { sp. }\end{array}$ \\
\hline $\begin{array}{l}\text { YB3 } \\
* *\end{array}$ & White & $\begin{array}{l}\text { White, } \\
\text { spreading }\end{array}$ & Velvety & Flat & $\sqrt{ }$ & Conidia & Ellipsoid & $\begin{array}{l}\text { Septate, } \\
\text { aerial }\end{array}$ & $\begin{array}{l}\text { Conidiophores hyalin, erect, bearing spore masses on } \\
\text { phialides at the apex: phialides verticilate }\end{array}$ & Gliocladium sp. \\
\hline $\begin{array}{l}\text { YB4 } \\
* *\end{array}$ & White & Yellowish white & Cottony & Umbonate & - & $\begin{array}{l}\text { Sporangiospore } \\
\text { hialin }\end{array}$ & Ellipsoid & $\begin{array}{l}\text { Septate, } \\
\text { aerial }\end{array}$ & $\begin{array}{l}\text { Sporangiophores hyaline, erect, branched sympodial, } \\
\text { sporangia terminal, rizblack, hitam, ovate }\end{array}$ & Mucor sp. \\
\hline $\begin{array}{l}\text { YB5 } \\
* *\end{array}$ & White & White & Cottony & Umbonate & $\sqrt{ }$ & Conidia & Globose & $\begin{array}{l}\text { Septate, } \\
\text { aerial }\end{array}$ & $\begin{array}{l}\text { Conidiophores dark brown, erect, bearing spore } \\
\text { masses in apical part nodes } 4 \text { positions. } \\
\text { Botryoblastosporous type }\end{array}$ & Gonatobotrys sp. \\
\hline
\end{tabular}




\begin{tabular}{|c|c|c|c|c|c|c|c|c|c|c|}
\hline Y2B & White & White & Cottony & Flat & $\sqrt{ }$ & Conidia & $\begin{array}{l}\text { Sub- } \\
\text { globose }\end{array}$ & $\begin{array}{l}\text { Septate, } \\
\text { aerial }\end{array}$ & $\begin{array}{l}\text { Hyalin conidiophore, phialides have apical spore } \\
\text { masses. Fialosforus conidia, clamidospores light } \\
\text { brown, subglobose. }\end{array}$ & Trichoderma sp. \\
\hline $\begin{array}{l}\text { Y2B6 } \\
* *\end{array}$ & $\begin{array}{l}\text { Yellowish } \\
\text { white }\end{array}$ & white & Cottony & Flat & $\sqrt{ }$ & Conidia & $\begin{array}{l}\text { Sub } \\
\text { Globose }\end{array}$ & Septate & $\begin{array}{l}\text { Hyalin conidiophore, phialides have apical spore } \\
\text { masses. Fialosforus conidia, clamidospores light } \\
\text { brown, subglobose. }\end{array}$ & Trichoderma sp. \\
\hline $\begin{array}{l}\text { YA1 } \\
* * * *\end{array}$ & $\begin{array}{l}\text { Yellowish } \\
\text { white }\end{array}$ & $\begin{array}{l}\text { White, } \\
\text { spreading }\end{array}$ & Cottony & Flat & $\sqrt{ }$ & Conidia & $\begin{array}{l}\text { Sub- } \\
\text { globose }\end{array}$ & $\begin{array}{l}\text { Septate, } \\
\text { aerial }\end{array}$ & $\begin{array}{l}\text { Hyalin conidiophore, phialides have apical spore } \\
\text { masses. Fialosforus conidia, clamidospores light } \\
\text { brown, subglobose. }\end{array}$ & Trichoderma sp. \\
\hline $\begin{array}{l}\text { YA2 } \\
* * *\end{array}$ & White & $\begin{array}{l}\text { White, } \\
\text { spreading }\end{array}$ & Cottony & Flat & $\sqrt{ }$ & Conidia & $\begin{array}{l}\text { Sub- } \\
\text { globose }\end{array}$ & $\begin{array}{l}\text { Septate, } \\
\text { non aerial }\end{array}$ & $\begin{array}{l}\text { Hyalin conidiophore, phialides have apical spore } \\
\text { masses. Fialosforus conidia, clamidospores light } \\
\text { brown, subglobose. }\end{array}$ & Trichoderma sp. \\
\hline $\begin{array}{l}\text { YA3 } \\
* * *\end{array}$ & White & $\begin{array}{l}\text { White, } \\
\text { spreading }\end{array}$ & Cottony & Flat & $\sqrt{ }$ & Conidia & $\begin{array}{l}\text { Sub- } \\
\text { globose }\end{array}$ & $\begin{array}{l}\text { Septate, } \\
\text { aerial }\end{array}$ & $\begin{array}{l}\text { Hyalin conidiophore, phialides have apical spore } \\
\text { masses. Fialosforus conidia, clamidospores light } \\
\text { brown, subglobose. }\end{array}$ & Trichoderma sp. \\
\hline $\begin{array}{l}\text { YA4 } \\
* * *\end{array}$ & $\begin{array}{l}\text { White, dark } \\
\text { centred }\end{array}$ & $\begin{array}{l}\text { White, } \\
\text { spreading }\end{array}$ & Cottony & Flat & - & Sporangiospore & Globose & $\begin{array}{l}\text { Septate, } \\
\text { aerial }\end{array}$ & $\begin{array}{l}\text { Sporangiophores erect, } 1-3 \text { branches, tapering } \\
\text { gradually towards the apex. Sporangia non columella }\end{array}$ & Mortierella sp. \\
\hline $\begin{array}{l}\text { YA5 } \\
* * *\end{array}$ & $\begin{array}{l}\text { White, dark } \\
\text { centred }\end{array}$ & Light yellow & Glaborous & Rugose & $\sqrt{ }$ & Clamydospore & Globose & $\begin{array}{l}\text { Septate, } \\
\text { aerial }\end{array}$ & $\begin{array}{l}\text { Sporangiophores hyaline, erect, branched, septate near } \\
\text { sporangia, rhizoidal basal. Sporangia globose, gray, } \\
\text { columellate, and conspicuous apophysate. }\end{array}$ & Gongronella sp. \\
\hline $\begin{array}{l}\mathrm{Y} 2 \mathrm{~A} 2 \\
* * *\end{array}$ & $\begin{array}{l}\text { White, dark } \\
\text { centred }\end{array}$ & $\begin{array}{l}\text { White, } \\
\text { spreading }\end{array}$ & Cottony & Flat & $\sqrt{ }$ & Conidia & $\begin{array}{l}\text { Ovate, } \\
\text { ellipsoid }\end{array}$ & $\begin{array}{l}\text { Septate, } \\
\text { aerial }\end{array}$ & $\begin{array}{l}\text { Hyalin conidiophore, phialides have apical spore } \\
\text { masses. Fialosforus conidia, clamidospores light } \\
\text { brown, subglobose. }\end{array}$ & $\begin{array}{l}\text { Wiesneriomyces } \\
\text { sp. }\end{array}$ \\
\hline
\end{tabular}


Table 2. Percentage antibacterial activity of Syzygium malaccense leaf extract and it endophytic fungal extract (at a concentration of 400 $\mu \mathrm{g} / \mathrm{disk})$ compared standard antibiotic tetracycline ( $30 \mu \mathrm{g} / \mathrm{disk})$

\begin{tabular}{llcccc}
\hline \multirow{2}{*}{ Sampels } & \multirow{2}{*}{ Extract } & \multicolumn{4}{c}{ Antibacterial activity ${ }^{\text {a }}$} \\
\cline { 3 - 5 } Leaves Syzygium malaccense & Methanol & $18.3 \pm 1.2$ & $16.5 \pm 1.7$ & $17.4 \pm 1.3$ & $17.5 \pm 2.3$ \\
endophytic fungi & YD1 & $7.5 \pm 1.2$ & $7.6 \pm 1.9$ & $9.4 \pm 0.8$ & $7.3 \pm 1.9$ \\
& YD2 & $12.7 \pm 1.7$ & $10.7 \pm 1.4$ & $12.9 \pm 2.1$ & $11.0 \pm 2.2$ \\
& YD3 & $17.5 \pm 2.5$ & $17.5 \pm 1.6$ & $11.1 \pm 1.9$ & $16.8 \pm 2.8$ \\
& YD4 & $9.9 \pm 1.2$ & $8.0 \pm 1.7$ & $8.4 \pm 1.3$ & $10.6 \pm 1.4$ \\
& YD6 & $10.2 \pm 1.4$ & $10.8 \pm 1.6$ & $13.5 \pm 1.8$ & $12.5 \pm 1.8$ \\
& YD7 & $7.9 \pm 2.1$ & $7.4 \pm 1.8$ & $8.5 \pm 1.3$ & $7.6 \pm 2.2$ \\
& Y2D1 & $18.9 \pm 2.3$ & $16.7 \pm 1.2$ & $16.8 \pm 1.5$ & $17.2 \pm 2.3$ \\
Stem bark Syzygium malaccense & Methanol & $17.9 \pm 1.8$ & $17.2 \pm 1.2$ & $17.4 \pm 1.4$ & $16.7 \pm 1.7$ \\
endophytic fungi & YB1 & $17.1 \pm 1.5$ & $15.8 \pm 1.7$ & $16.2 \pm 1.8$ & $17.3 \pm 1.5$ \\
& YB2 & $8.3 \pm 1.2$ & $7.7 \pm 2.2$ & $7.2 \pm 0.8$ & $8.2 \pm 2.2$ \\
& YB3 & $18.2 \pm 1.3$ & $16.2 \pm 1.4$ & $15.7 \pm 1.2$ & $15.7 \pm 1.2$ \\
& YB4 & $13.6 \pm 1.6$ & $11.2 \pm 1.8$ & $10.7 \pm 1.8$ & $13.6 \pm 0.9$ \\
& YB5 & $12.4 \pm 2.2$ & $11.7 \pm 1,3$ & $11.4 \pm 2.5$ & $6.6 \pm 2.3$ \\
& Y2B5 & $9.1 \pm 2.1$ & $11.8 \pm 0.83$ & $9.3 \pm 0.9$ & $8.9 \pm 2.9$ \\
& Y2B6 & $10.6 \pm 2,17$ & $11.7 \pm 0,83$ & $10.5 \pm 1.1$ & $9.7 \pm 2,91$ \\
Root bark Syzygium malaccense & Methanol & $16.3 \pm 1.1$ & $17.2 \pm 1.1$ & $17.5 \pm 1.6$ & $16.7 \pm 0.7$ \\
endophytic fungi & YA1 & $13.6 \pm 2.1$ & $15,9 \pm 2.6$ & $12.8 \pm 2.4$ & $15.9 \pm 3.1$ \\
& YA2 & $9.00 \pm 1.6$ & $9.7 \pm 1.4$ & $8.5 \pm 1.3$ & $8.3 \pm 1.8$ \\
& YA3 & $14.6 \pm 1.1$ & $16.3 \pm 1.6$ & $15.9 \pm 1.4$ & $14.4 \pm 2.1$ \\
Antibiotic ${ }^{b}$ & YA4 & $16.6 \pm 1.3$ & $15.2 \pm 1.7$ & $16.9 \pm 1.9$ & $15.6 \pm 1.5$ \\
\hline YA5 & $17.2 \pm 1.4$ & $15.9 \pm 2.5$ & $15.9 \pm 1.9$ & $15.1 \pm 1.8$ \\
& Y2A2 & $11.7 \pm 1.6$ & $15.7 \pm 1.4$ & $11.2 \pm 2.4$ & $12.6 \pm 0.8$ \\
& Tetracycline & $22.2 \pm 1.14$ & $20.5 \pm 1.32$ & $20.7 \pm 1.22$ & $21.6 \pm 1.25$ \\
\hline
\end{tabular}

Note: a. Percentage of antibacterial activity extract compared to standard antibiotics (\%): zone of inhibition (mm) extract/ zone of inhibition $(\mathrm{mm})$ tetracycline: $* * *$ strong $(\geq 70 \%), * *$ moderate $(50-70 \%)$, and $*_{\text {weak }}(<50 \%)$ against Salmonella typhi, Bacillus subtilis, Escherichia coli, and Staphylococcus aureus, b. positive control (30 g/disk), (-) no inhibition zone

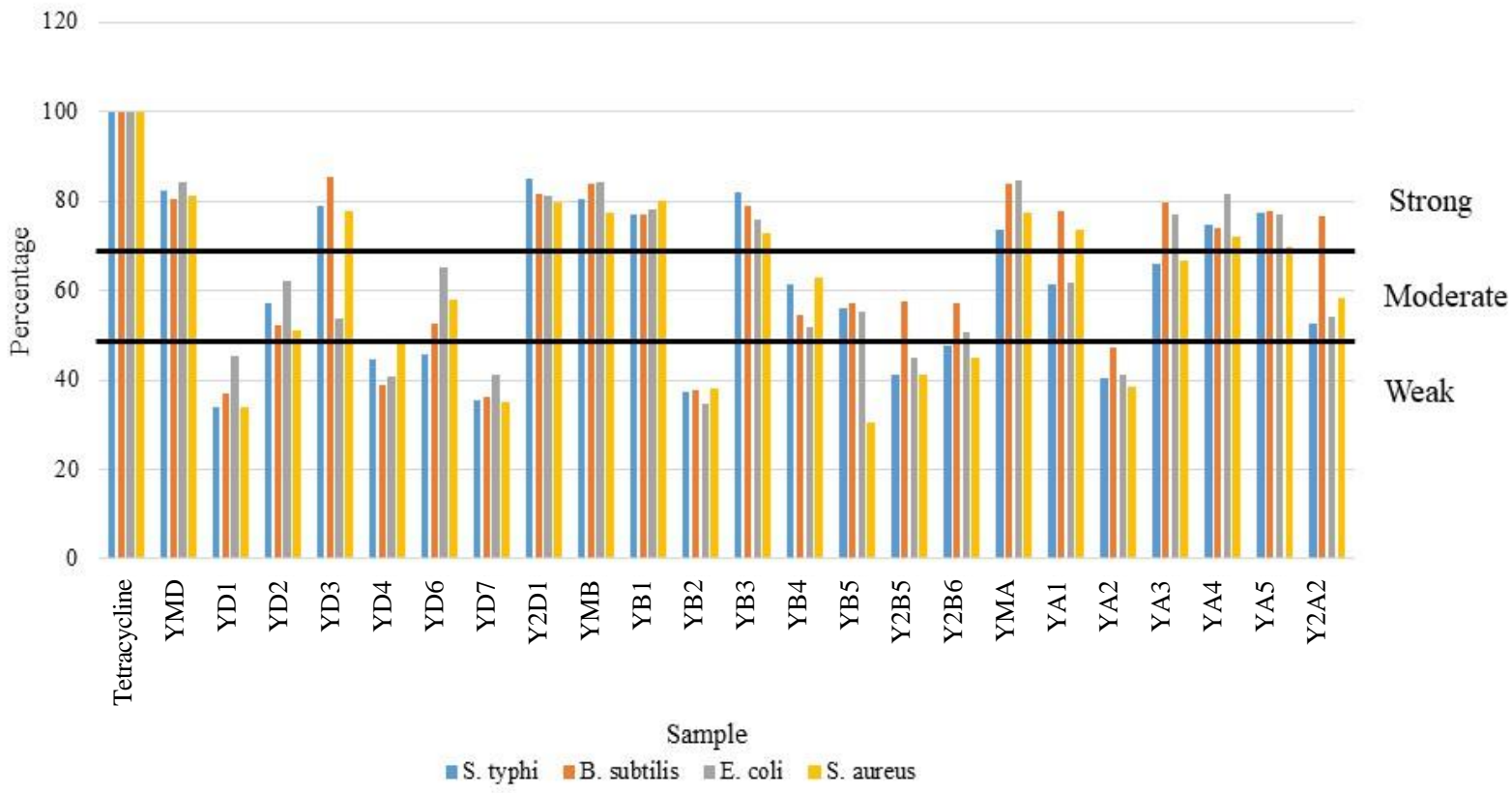

Figure 3. Antibacterial activity of Syzygium malaccense leaf methanol extract and its endophytic fungus extract (concentration 400 $\mu \mathrm{g} / \mathrm{mL}$ ) as a percentage of strength against Salmonella typhi, Bacillus subtilis, Escherichia coli, and Staphylococcus aureus in comparison to tetracycline $(30 \mu \mathrm{g} / \mathrm{mL})$ 
Table 3. Diversity of endophytic fungi isolated from Syzygium malaccense

\begin{tabular}{lcccc}
\hline \multirow{2}{*}{ Genera } & \multicolumn{5}{c}{ Part of plat Syzygium malaccense } \\
\cline { 2 - 5 } & Leave & $\begin{array}{c}\text { Stem } \\
\text { bark }\end{array}$ & $\begin{array}{c}\text { Root } \\
\text { bark }\end{array}$ & Total \\
\hline Poaceascoma & 1 & 0 & 0 & 1 \\
Cladorrhinum & 1 & 0 & 0 & 1 \\
Penicillium & 1 & 0 & 0 & 1 \\
Madurella & 1 & 0 & 0 & 1 \\
Phytophthora & 1 & 0 & 0 & 1 \\
Phialemonium & 1 & 0 & 0 & 1 \\
Monascus & 1 & 0 & 0 & 1 \\
Trichoderma & 0 & 3 & 3 & 6 \\
Ramophialophora & 0 & 1 & 0 & 1 \\
Gliocladium & 0 & 1 & 0 & 1 \\
Mucor & 0 & 1 & 0 & 1 \\
Mortierella & 0 & 0 & 1 & 1 \\
Gongronella & 0 & 0 & 1 & 1 \\
Gonatobotrys & 0 & 1 & 0 & 1 \\
Wiesneriomyces & 0 & 0 & 1 & 1 \\
Jumlah isolat fungi & 7 & 7 & 6 & 20 \\
Simpson index (D) & 0.1429 & 0.2653 & 0.3333 & 0.125 \\
Simpson index of & 0.8571 & 0.7347 & 0.6667 & 0.875 \\
diversity (1-D) & & & & \\
Shannon Index of & 1.946 & 1.475 & 1.242 & 2.458 \\
diversity (H') & & & & \\
\hline
\end{tabular}

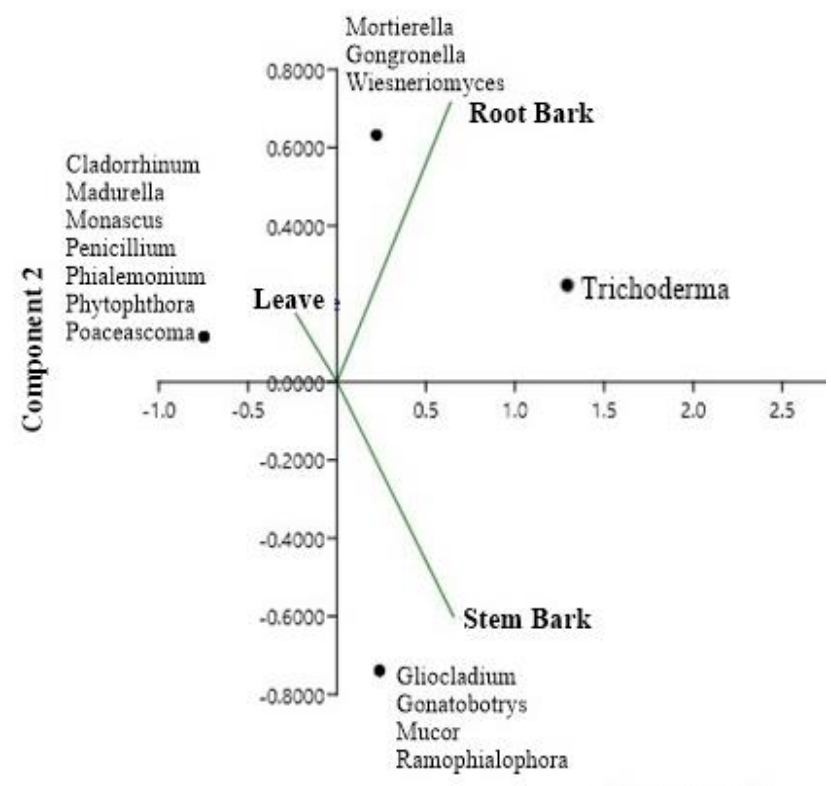

Component 1

Figure 4. PCA (Principal Component Analisis) Syzygium malaccense endophytic fungi isolates in different organs. In components 1 and 2 have a variation of $60 \%$ diversity

In Figure 4 and Table 3, isolates of the same endophytic fungus, Trichoderma sp., apparently of the same species, were isolated from stem bark and root bark isolates of $S$. malaccense. Trichoderma can be found in a number of settings and seasons. This fungus genus contains a diverse range of species that have been isolated from soils, acidic soils, bark, varied substrates, and tree bark. Trichoderma is distinguished by the shape of its conidia, which can range from globose to ellipsoidal, obovoidal, or short cylindrical with a truncate and tapering basal tip. Smooth to roughwalled, wing-like projections, obovoid, oval, or shortly cylindrical, sub-globose single-celled conidia, colorless, green, gray, or brownish; smooth to rough-walled, wing-like projections, may Intercalary or terminal chlamydospores, elliptical circular, greenish, yellowish, or colorless (Misra and Gupta 2009; Sun et al. 2012; Sharma et al. 2019).

\section{Discussion}

Endophytic fungus isolated from the leaves, stem bark, and root bark of S. malaccense plants from Palembang, South Sumatra, produced various antibacterial substances depending on their taxonomic groupings. These three plant parts could have varied functions in supporting host growth and nutritional needs in pathogen protection (Selim et al. 2012; Fitriarni and Kasiamdari 2018). Endophytic fungi create secondary compounds that protect host plants against pathogens, consequently, plant parts can produce a variety of endophytic fungal isolates (Fadiji and Babalola 2020). Endophytic fungi on leaves were mainly found in the lamina, and colonization increased during the rainy season (Chutulo and Chalannavar 2018). Several factors, including ecology, nutrition, host physiology, and fungal colonization sites, are thought to be correlated with the establishment and amount of secondary metabolites produced by endophytic fungi (Chauhan et al. 2019).

In the antibacterial activity test at a $400 \mu \mathrm{g} / \mathrm{mL}$ concentration, S. typhi, B. subtilis, E. coli, and S. aureus bacteria were inhibited by the ethyl acetate extract of endophytic fungi isolates and plant extracts from $S$. malaccense. This result is presumably because endophytic fungi can form the same secondary metabolites as their hosts (Zhao et al. 2010; Jia et al. 2016; Pelo and Mavumengwana 2020). Endophytic fungi are believed to imitate, duplicate, and modify the secondary metabolite compounds of their hosts, which is a complex process (Gouda et al. 2016). The group of fungi that had a substantial percentage of antibacterial activity as their host was found in the leaf methanol extract and fungi extract Y2D1. Still, antibacterial activities were observed in stem bark methanol extracts, such as YB1 and YB3, and fungi extracts from root bark methanol extract, such as fungi extract YA4 and YA5. Natural products derived from endophytic fungi are considered the most relevant discovery sources and molecular diversity for new drugs. It was shown in several studies that most bioactive metabolites obtained from endophytic fungi have unique chemical structures. These fungi are capable of producing metabolites similar to those produced by their hosts or different new compounds. This represents a substantial biotechnological potential of endophytic fungi for the discovery of new drugs (Cruz et al. 2020; El-Hawary et al. 2020). Endophytic fungi can duplicate and modify compounds produced by their host plants. Endophytic fungi can duplicate and change compounds by making them more complex and increasing their function (Verma et al. 2009). 
There are some secondary metabolites (e.g., phenolics, flavonoids, and carotenoids) in the different parts of $S$. malaccense, significantly in its highest leaves, which are helpful as nutrition and pharmacology substances (Batista et al. 2017). Secondary metabolites are hypothesized to work as antibacterial substances in host plants and endophytic fungi by preventing cell wall development, modifying membrane permeability, inhibiting nucleic acid production, and decreasing the formation of essential metabolites in bacterial cells (Chatterjee et al. 2019). With the results of macroscopic and microscopic identification in Figure 3 (YD3 and Y2D1), (YB1 and YB3), and (YA4 and YA5), it is suggested that endophytic fungal isolates are antibacterial agents from the Ascomycota and Zygomycota phyla. In the Zygomycota phylum, the obtained genera were Mucor, Mortierella, and Gongronella. Regarding the Ascomycota phylum, the obtained genera were Monascus, Penicillium, Gliocladium, and Trichoderma, including isolate Y2D1 from the genus Monascus, which was the best group of endophytic fungal isolates that could suppress the tested bacteria. It is thought that the species from the Monascus genus produce monascidin A, a substance with antibacterial activities that inhibit bacterial and fungal strains (Ferdeş et al. 2004). The species Monascus purpuresus is a multicolored fungus with several biological properties, including antibacterial activities (Bi and Gajalakshmi 2018). Penicillium species produce more than 280 chemicals with antibacterial, anticancer, antiviral, antioxidant, anti-inflammatory, antiparasitic, immunosuppressive, antidiabetic, antiobesity, antifibrotic, neuroprotective, insecticidal, and biocontrol properties (Toghueo and Boyom 2020). Under microaerophilic circumstances, the species from the Gliocladium genus may break down plant cellulose, generating complex hydrocarbons (Ahamed and Ahring 2011). The fungi species from the genus Trichoderma live in the soil, potentially forming endophytic relationships with host plants that protect them from harmful fungi (Ruangwong et al. 2021). As an endophytic fungus, species from the Mortierella genus aid plants in absorbing $\mathrm{P}$ and $\mathrm{Fe}$ elements and protecting host plants from infections. (Ozimek and Hanaka 2021).

In methanol extracts of host plant tissues and endophytic fungi isolated from leaves, stem bark, and root bark, S. malaccense secondary metabolites had antibacterial activity. Furthermore, the species of endophytic fungi and their compounds from these potential secondary metabolites must be investigated.

\section{ACKNOWLEDGEMENTS}

The authors thank to the DRPM, Ministry of Research and Technology, Indonesia, which provided research funding through Hibah Disertasi Doktor 2021, with contract no. 0166.04/UN9/SB3.LP2M.PT/2021

\section{REFERENCES}

Abdalla MA, McGaw LJ. 2018. Bioprospecting of South African plants as a unique resource for bioactive endophytic microbes. Front Pharmacol 9: 456. DOI: 10.3389/fphar.2018.00456.

Ahamed A, Ahring BK. 2011. Production of hydrocarbon compounds by endophytic fungi Gliocladium species grown on cellulose. Bioresour Technol 102 (20): 9718-9722. DOI: 10.1016/j.biortech.2011.07.073.

Arzamani K, Vatandoost H, Rassi Y, Akhavan AA, Abai MR, Alavinia M, Akbarzadeh K, Mohebali M, Rafizadeh S. 2018. Richness and diversity of phlebotomine sand flies (Diptera: Psychodidae) in North Khorasan Province, Northeast of Iran. J Arthropod-Borne Dis 12 (3): 232. DOI: $10.18502 /$ jad.v12i3.75.

Bairy K, Sharma A, Shalini, Adiga. 2005. Evaluation of the hypoglycemic, hypolipidemic and hepatic glycogen raising effects of Syzygium malaccense upon streptozotocin induced diabetic rats. J Nat Remedies 5 (1): 46-51.

Batista ÂG, da Silva JK, Cazarin CB, Biasoto AC, Sawaya AC, Prado MA, Júnior MR. 2017. Red-jambo (Syzygium malaccense): Bioactive compounds in fruits and leaves. LWT - Food Sci Technol 76: 284291. DOI: $10.1016 /$ j.lwt.2016.05.013.

Bi MA, Gajalakshmi P. 2018. Antibacterial and antioxidant activity of the pigment produced by Monascus purpureus. Eurasian J Anal Chem 13 (2): 378-384

Chatterjee S, Ghosh R, Mandal NC. 2019. Production of bioactive compounds with bactericidal and antioxidant potential by endophytic fungus Alternaria alternata AE1 isolated from Azadirachta indica A. Juss. Plos One 14 (4): e0214744.

Chauhan NM, Gutama AD, Aysa A. 2019. Endophytic fungal diversity isolated from different agro-ecosystem of Enset (Ensete ventericosum) in Gedeo zone, SNNPRS, Ethiopia. BMC Microbiol 19 (1): 1-10. DOI: 10.1186/s12866-019-1547-y.

Chutulo EC, Chalannavar RK. 2018. Endophytic mycoflora and their bioactive compounds from Azadirachta indica: A comprehensive review. J Fungi 4 (2): 42. DOI: 10.3390/jof4020042.

Cruz J, da Silva C, Hamerski L. 2020. Natural products from endophytic fungi associated with Rubiaceae species. J Fungi 6 (3): 1-26. DOI: 10.3390/jof6030128.

da Silva Dantas CC, de Souza EL, Cardoso JD, de Lima LA, de Sousa Oliveira K, Migliolo L, Dias SC, Franco OL, Magnani M. 2014. Identification of a napin-like peptide from Eugenia malaccensis L. seeds with inhibitory activity toward Staphylococcus aureus and Salmonella enteritidis. Protein J 33 (6): 549-556. DOI: 10.1007/s10930-014-9587-5.

Ding N, Chang X, Shi N, Yin X, Qi F, Sun Y. 2019. Enhanced inactivation of antibiotic-resistant bacteria isolated from secondary effluents by gC 3 N 4 photocatalysis. Environ Sci Pollut Res 26 (18): 18730-18738. DOI: 10.1007/s11356-019-05080-7.

Du W, Yao Z, Li J, Sun C, Xia J, Wang B, Shi D, Ren L. 2020. Diversity and antimicrobial activity of endophytic fungi isolated from Securinega suffruticosa in the Yellow River Delta. PloS One 15 (3): e0229589. DOI: 10.1371/journal.pone.0229589.

El-Bondkly EAM, El-Bondkly AAM, El-Bondkly AAM. 2021. Marine endophytic fungal metabolites: A whole new world of pharmaceutical $\begin{array}{lllll}\text { therapy exploration. Heliyon } 7 \text { (3): e06362. DOI: } & \end{array}$ 10.1016/j.heliyon.2021.e06362.

El-hawary SS, Moawad AS, Bahr HS, Abdelmohsen UR, Mohammed R. 2020. Natural product diversity from the endophytic fungi of the genus Aspergillus. RSC Adv 10 (37): 22058-22079. DOI: 10.1039/d0ra04290k.

Fadhillah F, Yohandini H, Widjajanti H. 2019. Chemical compound isolated from antioxidant active extract of endophytic fungus Cladosporium tenuissimum in Swietenia mahagoni leaf stalks. Biodiversitas 20 (9): 2645-2650. DOI: 10.13057/biodiv/d200929.

Fadiji AE, Babalola OO. 2020. Exploring the potentialities of beneficial endophytes for improved plant growth. Saudi J Biol Sci 27 (12): 3622-3633. DOI: 10.1016/j.sjbs.2020.08.002.

Ferdes M, Ungureanu C, Radu N, Chirvase AA. 2009. Antimicrobial effect of Monascus purpureus red rice against some bacterial and fungal strains. New Biotechnol 25: $1 . \quad$ DOI: 10.1016/j.nbt.2009.06.119. 
Fernandes FA, Rodrigues S. 2018. Jambo-Syzygium malaccense. In Exotic Fruits. Academic Press. DOI: 10.1016/B978-0-12-803138-4.00031-9.

Fitriarni D, Kasiamdari RS. 2018. Isolation and identification of endophytic fungi from leave and stem of Calopogonium mucunoides. J Trop Biodivers Biotechnol 3 (1): 30-36. DOI: 10.22146/jtbb.32477.

Freitas T, Pereira L, Pereira C. 2015. Syzygium sp (Myrtaceae): Promising for diabetes treating? Eur J Med Plants 7 (4): 167-176. DOI 10.9734/ejmp/2015/14592.

Gouda S, Das G, Sen SK, Shin HS, Patra JK. 2016. Endophytes: a treasure house of bioactive compounds of medicinal importance. Front Microbiol 7: 1538. DOI: 10.3389/fmicb.2016.01538

Habisukan UH, Elfita E, Widjajanti H, Setiawan A. 2021. Diversity of endophytic fungi in Syzygium aqueum. Biodivers J Biol Divers 22 (3): 1129-1137. DOI: 10.13057/biodiv/d220307.

Jia M, Chen L, Xin HL, Zheng CJ, Rahman K, Han T, Qin LP. 2016. A friendly relationship between endophytic fungi and medicinal plants: A systematic review. Front Microbiol 7 (JUN): 906. DOI 10.3389/fmicb.2016.00906

Locher CP, Burch MT, Mower HF, Berestecky J, Davis H, Van Poel B, Lasure A, Berghe DV, Vlietinck AJ. 1995. Anti-microbial activity and anti-complement activity of extracts obtained from selected Hawaiian medicinal plants. J Ethnopharmacol 49 (1): 23-32. DOI: 10.1016/0378-8741(95)01299-0.

Manganyi MC, Ateba CN. 2020. Untapped potentials of endophytic fungi: A review of novel bioactive compounds with biological applications. Microorganisms 8 (12): 1-25. DOI: 10.3390/microorganisms8121934.

Metasari S, Elfita, Muharni, Yohandini H. 2020. Antioxidant compounds from the stem bark of Syzygium samarangense L. Molekul 15 (3): 175-183. DOI: 10.20884/1.jm.2020.15.3.626.

Misra AK, Gupta VK. 2009. Trichoderma: Biology, biotechnology and biodiversity. J Eco-Friendly Agric 4 (2): 99-117.

Nawaz H, Shad MA, Rehman N, Andaleeb H, Ullah N. 2020. Effect of solvent polarity on extraction yield and antioxidant properties of phytochemicals from bean (Phaseolus vulgaris) seeds. Braz J Pharm Sci 56. DOI: 10.1590/s2175-97902019000417129.

Oyinlade OC. 2014. Phytochemical and physicochemical analysis of three different types of apples. Intl J Sci Res Rev 3 (1): 67-78

Ozimek E, Hanaka A. 2021. Mortierella species as the plant growthpromoting fungi present in the agricultural soils. Agriculture (Switzerland) 11 (1): 1-18. DOI: 10.3390/agriculture11010007.

Pelo S, Mavumengwana V. 2020. Diversity and antimicrobial activity of culturable fungal endophytes in Solanum mauritianum. Intl J Environ Res Publ Health 17 (2): 439. DOI: 10.3390/ijerph17020439.

Pitt JI, Hocking AD. 2009. Fungi and Food Spoilage (Third Edit). Springer, New York. DOI: 10.1007/978-0-387-92207-2 1.
Ruangwong OU, Wonglom P, Suwannarach N, Kumla J, Thaochan N, Chomnunti P, Pitija K, Sunpapao A. 2021. Volatile organic compound from Trichoderma asperelloides TSU1: Impact on plant pathogenic fungi. J Fungi 7 (3): 187. DOI: 10.3390/jof7030187.

Selim KA, El-Beih AA, AbdEl-Rahman TM, El-Diwany AI. 2012. Biology of endophytic fungi. Curr Res Environ Appl Mycol 2 (1): 3182. DOI: $10.5943 / \mathrm{cream} / 2 / 1 / 3$.

Sharma S, Kour D, Rana KL, Dhiman A, Thakur S, Thakur P, Thakur S, Thakur N, Sudheer S, Yadav N, Yadav AN. 2019. Trichoderma: biodiversity, ecological significances, and industrial applications. In Recent advancement in white biotechnology through fungi. Springer, Cham. DOI: 10.1007/978-3-030-10480-1_3.

Singh A, Singh DK, Kharwar RN, White JF, Gond SK. 2021. Fungal endophytes as efficient sources of plant-derived bioactive compounds and their prospective applications in natural product drug discovery: Insights, avenues, and challenges. Microorganisms 9 (1): 197. DOI: 10.3390/microorganisms9010197.

Sonavane P. 2017. First report of Collectotrichum acutatum J.H simmonds associated with anthracnose disease in malayan apple from India. Intl J Pure Appl Biosci 5 (5): 465-472. DOI: 10.18782/23207051.2712 .

Strobel G, Daisy B. 2003. Bioprospecting for microbial endophytes and their natural products. Microbiol Mol Biol Rev 67 (4): 491-502. DOI: 10.1128/mmbr.67.4.491-502.2003.

Sun RY, Liu ZC, Fu K, Fan L, Chen J. 2012. Trichoderma biodiversity in China. J Appl Genet 53 (3): 343-354. DOI: 10.1007/s13353-0120093-1.

Toghueo RM, Boyom FF. 2020. Endophytic Penicillium species and their agricultural, biotechnological, and pharmaceutical applications. 3 Biotech 10 (3): 1-35. DOI: 10.1007/s13205-020-2081-1.

Verma VC, Kharwar RN, Strobel GA. 2009. Chemical and functional diversity of natural products from plant associated endophytic fungi. Nat Prod Commun 4 (11): 1934578X0900401114. DOI: $10.1177 / 1934578$ X0900401114.

Walsh TJ, Hayden RT, Larone DH. 2018. Larone's Medically Important Fungi: A Guide to Identification. John Wiley \& Sons. DOI: 10.1128/9781555819880.

Watanabe T. 2010. Pictorial atlas of soil and seed fungi: Morphologies of cultured fungi and key to species. CRC Press/Taylor \& Francis. DOI: 10.1201/ebk1439804193.

Zhao J, Zhou L, Wang J, Shan T, Zhong L, Liu X, Gao X. 2010. Endophytic fungi for producing bioactive compounds originally from their host plants. Curr Res, Technol Educ Trop Appl Microbiol Microbial Biotechnol 1: 567-576. 